\title{
Differentiation of Active and Passive Response to Poliomyelitis Vaccine
}

\author{
By \\ Gordon C. Brown*
}

Although $\mathrm{I}$ intend to make this presentation as brief as possible, I would like to have the opportunity to comment on a point made by Dr. Evans with reference to the possible necessity for developing new vaccines against the enteroviruses. I agree with him almost 100 per cent when he mentions that many of the enteroviruses other than poliomyelitis cause only a relatively mild form of disease. However, I would like to point out that in the last large epidemic of poliomyelitis which occurred in Michigan in 1958, we made both virological and serological studies on over 1,000 patients with particular reference to those patients who had received a full course of inactivated poliomyelitis vaccine. Many of the clinical illnesses which were diagnosed as poliomyelitis were paralytic and some of them were fatal. Laboratory studies, however, showed that some of these serious illnesses including deaths were actually caused by Coxsackie type B5 and by ECHO 9 viruses. Although this information is not new, I think it is well to keep in mind that some of these other enteroviruses can cause quite serious clinical illness. An additional fundamental serious consequence of infection with Coxsackie virus results when pregnant women or their newborn babies are infected with type B viruses, especially late in pregnancy. These agents are frequently the cause of neonatal death in the young infant and in spite of this serious consequence the mother usually shows only very mild symptoms such as a slight upper respiratory infection with elevation of temperature. In fact, we recently had two such cases in less than one month. It therefore seems to me that one of the next targets for immunization, in an effort to save the lives of young children, is to immunize pregnant women or women of childbearing age against the Coxsackie B viruses. I am sorry for this digression but felt that these points were sufficiently important to comment on.

* Professor, Department of Epidemiology, School of Public Health, University of Michigan, Ann Arbor, Michigan. 
My immediate concern has to do with one of the fundamental points mentioned by Dr. David Evans, that of the necessity for assuring ourselves of an adequate response to a vaccine if we are going to use it. Our interest has been with the killed rather than the live vaccine particularly because we are interested in combinations of killed virus and bacterial vaccines. Such combinations, of course, would be very difficult, if not impossible, to perfect if one used live agents because of the phenomenon of interference between organisms. We have done extensive studies with inactivated quadruple vaccines containing the antigens of diphtheria, pertussis, tetanus, and the three types of poliomyelitis and at the present time are investigating a combination of killed measles and polio vaccines. However, we are still concerned with the problem of determining the best time to give the vaccine and with the best immunization schedule in order to assure the most likely satisfactory response in the young infant. Such a satisfactory response is complicated by the fact that high titers of maternal antibody can suppress it in a large number of infants. Dr. Perkins here in the audience has studied this extensively in England as has Dr. Wilson in Canada and several others in our country have also discovered this phenomenon. The problem is even more pertinent in the United States where we have a situation which is apparently different from most of the world, namely, almost universal vaccination of pregnant women against poliomyelitis. If such women have been immunized within the past two or three years, they are still given one dose of vaccine by the obstetrician. If they have not had any previous poliomyelitis vaccine, they are given as many as three doses during pregnancy. This procedure is fine as far as the protection of the pregnant women is concerned but it also results in extremely high titers of poliomyelitis antibody at the time of delivery with the result that these antibodies are passively transferred to the infant in whom it is still detectable for several months. During this time if the passive antibody titer is $1: 16$ or greater, an active response to killed vaccine will be suppressed in a large number of children. Unless a pediatrician chooses to delay poliomyelitis immunization until the fifth or sixth month of life, and many do not, there is no guarantee of a satisfactory response. We have found, however, that when the primary series is given at an interval of two months instead of at an interval of one month, the response is better. This then leads to the problem to be discussed today; namely, is there any way of determining which infants have maternally transferred passive antibody and which ones have actively acquired antibody? A child or young infant may have neutralizing antibodies which are clearly detectable by conventional techniques but this does not differentiate whether they were actively or passively acquired. A possible means of differentiating these two types of antibody through fluorescent microscopy is herein presented. 
and 87 per cent detection of fluorescent antibodies. A passive response, on the other hand, although 100 per cent detectable by the neutralization technique, results in only 37 per cent detection by the FA technique. Thus, in approximately two-thirds of the individuals, passive antibodies can be differentiated from active antibodies when this technique is employed. The exact explanation for this phenomenon is not clear to us

Table 5. Differentiation of Active and Passive Response

\begin{tabular}{|c|c|c|c|c|c|}
\hline \multirow{3}{*}{$\begin{array}{c}\text { Prevaccine } \\
\text { Antibody } \\
\text { Titer }\end{array}$} & \multirow{3}{*}{$\begin{array}{c}\text { Type of } \\
\text { Response }\end{array}$} & \multicolumn{4}{|c|}{ Antibody Detected } \\
\hline & & \multicolumn{2}{|c|}{ Neut. } & \multicolumn{2}{|c|}{ F. A. } \\
\hline & & Prevacc. & Postrace. & Prevace. & Postvace. \\
\hline $\begin{array}{l}\text { None } \\
\text { Low }\end{array}$ & $\begin{array}{l}\text { active } \\
\text { active }\end{array}$ & $\begin{array}{r}0 / 29 \\
10 / 10\end{array}$ & $\begin{array}{l}28 / 29(96 \%) \\
10 / 10\end{array}$ & $\begin{array}{l}6 / 29(21 \%) \\
2 / 10(20 \%)\end{array}$ & $\begin{array}{r}25 / 29(86 \%) \\
9 / 10(90 \%)\end{array}$ \\
\hline & & $10 / 39(26 \%)$ & ${ }^{38} / 39(97 \%)$ & $8 / 89(21 \%)$ & ${ }^{34} / 39(87 \%)$ \\
\hline High & passive & ${ }^{38} / 38(100 \%)$ & ${ }^{38} / 38(100 \%)$ & ${ }^{14} / 38(37 \%)$ & ${ }^{14} / 38(37 \%)$ \\
\hline
\end{tabular}

at the present time, but investigation under progress in our laboratory is highly suggestive that the phenomenon is correlated with protein "decay" of the passive antibody which is undergoing degradation in the body of the young infant and therefore this antibody does not react with the final labeled antiserum which has been prepared against intact human gamma globulin. The possible signifieance of a laboratory technique to differentiate passive from actively acquired antibodies is great and may have far reaching consequences in the study of the response of young infants to active immunization procedures. 